\title{
Online unbalanced vibration suppression of a flexible rotor supported by active magnetic bearing
}

\author{
Huachun $\mathrm{Wu}^{1,2}$ \\ 1 School of Mechanical and Electronic Engineering, Wuhan University of Technology, Wuhan 430070, China; \\ whc@whut.edu.cn \\ 2 Hubei Provincial Engineering Technology Research Center for Magnetic Suspension, Wuhan 430070, \\ China
}

\begin{abstract}
A rotor supported by active magnetic bearing could offer various advantages over conventional bearings, but due to their limited force capacity, and moreover, the rotor has a mass unbalance. The vibration originated from the rotor's unbalance mass impacts on the dynamic properties, and even cause major accidents. Large unbalance is typically responsible for high energy vibrations and the consequent decrease in machine life. So unbalance compensation on the rotating machine is necessary, especially for the high speed flexible rotor. While the unbalanced vibration suppression need shutdown and phase sensor. To solve the problem of online suppress the unbalance vibration without adding additional hardware equipment in active magnetic bearingrotor system, this paper proposes an online unbalanced vibration suppression based on the crosscorrelation method and influence coefficient method. The amplitude and phase are achieved by cross-correlation method and feedback tracking control method in steady state. The active magnetic bearings are used to simulate the unbalanced excitation, the compensation amplitude of unbalance vibration signal are calculated by the influence coefficient method. The method was found to be effective and practical. An online test program was performed on a flexible rotor based on based on the Matlab/Simulink and a dSPACE real time system, which showed that the unbalanced vibration suppression could satisfy the performance and required precision of rotating machine, and the amplitude of the fundamental frequency of the unbalance vibration is significantly reduced.
\end{abstract}

Keywords: Active magnetic bearing; online unbalanced vibration suppression; influence coefficient method; cross-correlation method

\section{Introduction}

Nowadays, the rotating machinery has gradually developed from past low-power, lowefficiency, and low-speed to modern high-speed, high-precision, and high-efficiency. At the same time, the performance requirements of the rotating machinery are becoming higher and higher, mainly in high-speed, high-precision, high-speed reliability and adaptability to complex and harsh environments. Active Magnetic Bearings (AMB) which has no mechanical contact between rotor and stator can be found in some industry applications such as high speed rotor and turbomolecular pumps, e.g. ${ }^{[1,2]}$. However, due to uneven materials, processing accuracy, assembly errors, and other reasons, rotating machinery always has a certain unbalance even with very precise machining of rotor parts. It is difficult to avoid the mass unbalance. But due to their limited force capacity, and moreover, 
1st International Electronic Conference on Actuator Technology: Materials, Devices and Applications (IeCAT 2020) the rotor has a mass unbalance. The vibration originated from the rotor's unbalance mass impacts on the dynamic properties, and even cause major accidents. Large unbalance is typically responsible for high energy vibrations and the consequent decrease in machine life. So unbalance compensation on the rotating machine is necessary, especially for the high speed flexible rotor.

Generally, the rotor either spin around its geometric axis by counteracting the unbalance force or spin freely around its inertial axis by ignoring the synchronous component of rotor vibration signal, provided that the clearance between rotor and stator is sufficiently large ${ }^{[3,4,5]}$. So there have two control methods which are the unbalance compensation and the automation balancing. While the unbalanced vibration suppression need shutdown and phase sensor. How to solve the problem of online suppress the unbalance vibration without adding additional hardware equipment in active magnetic bearing-rotor system.

In this paper, an online unbalanced vibration suppression based on the cross-correlation method and influence coefficient method is presented for the flexible rotor supported by active magnetic bearing. The amplitude and phase are achieved by cross-correlation method and feedback tracking control method in steady state. The active magnetic bearings are used to simulate the unbalanced excitation, the compensation amplitude of unbalance vibration signal are calculated by the influence coefficient method. An online test program was performed on a flexible rotor based on based on Matlab/Simulink and a dSPACE real time system, which showed that the unbalance vibration suppression could satisfy the performance and required precision of rotating machine, and the amplitude of the fundamental frequency of the unbalance vibration is significantly reduced.

\section{Methods}

\subsection{Principle of the cross-correlation method}

The initial vibration signal caught by displacement sensors not only contains the fundamental frequency component, but also includes high-order harmonic, random noise, DC component, etc. Set the initial vibration signal as

$$
x(t)=x_{0}+A_{0} \sin \left(2 \pi f_{0} t+\varphi_{0}\right)+\sum_{i=1}^{n} A_{i} \sin \left(2 \pi f_{i} t+\varphi_{i}\right)+N(t)
$$

Where, $x_{0}$ represents DC component, $N(t)$ represents random noise, $A_{0} \sin \left(2 \pi f_{0} t+\varphi_{0}\right)$ is the fundamental frequency component.

The principle of cross-correlation analysis method is shown as following (Fig. 1) ${ }^{[4]}$

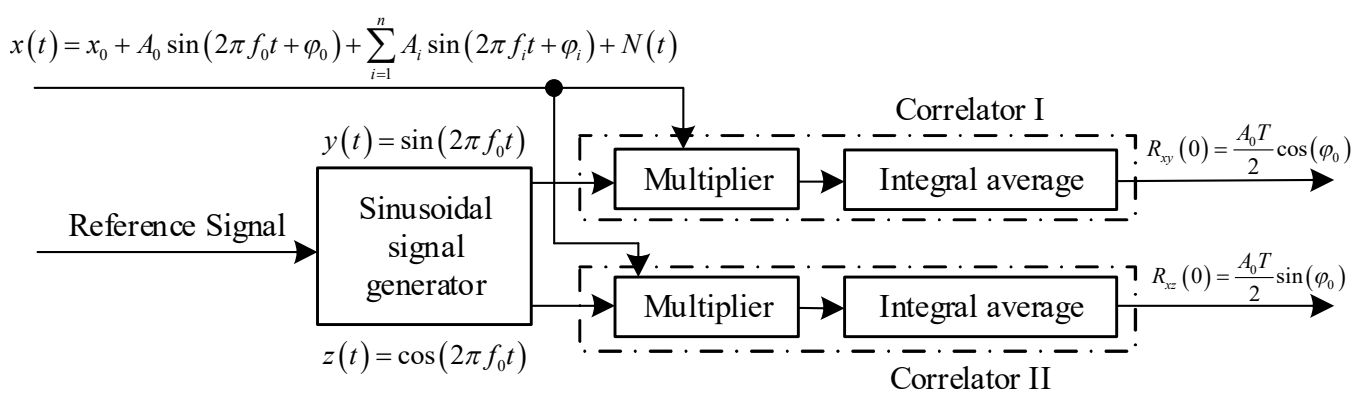

Figure 1. The principle of cross-correlation analysis.

$$
\left\{\begin{array}{l}
A_{0}=\frac{2 \sqrt{R_{x y}^{2}(0)+R_{x z}^{2}(0)}}{T} \\
\varphi_{0}=\arctan \frac{R_{x z}(0)}{R_{x y}(0)}
\end{array}\right.
$$


1st International Electronic Conference on Actuator Technology: Materials, Devices and Applications (IeCAT 2020)

When $R_{x y}(0)<0, \varphi=\pi+\varphi_{0}$; when $R_{x y}(0)>0$ and $R_{x z}(0)<0, \varphi=\pi+\varphi_{0}$.

\subsection{Principle of the influence coefficient method}

The influence coefficient method was adopted to calculate the value of the unbalance compensation, the key of influence coefficient method is to determine the influence coefficient matrix. The input of the system is the trial weight put on the correction planes; the output is the variation of the fundamental frequency signal of the rigid rotor under the same condition, the transitive relationship of input and output is the influence coefficient of the system ${ }^{[4]}$. It can be simulated unbalanced mass through electromagnetic force due to have two correction plane (at the left and right $\mathrm{AMB}$ ) and two measure plane (at the displacement sensor) in the active magnetic bearing-rotor system, as shown in Fig. 2.

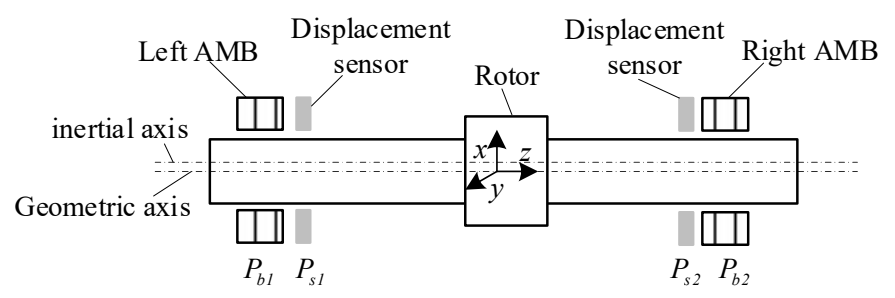

Figure 2. Active magnetic bearing-rotor system.

Assuming that the unbalanced force of the rotor is supported by the left and right radial magnetic bearings, so the original unbalanced force on the rotor can be equivalent to the positions of the two active magnetic bearings by using the equivalent principle [6,7]. Therefore, the aim of controlling the unbalanced force of the rotor system can be achieved by restraining the equivalent unbalanced force of bearing position.

By measuring the initial vibration displacement vectors $\vec{P}_{s 10}$ and $\vec{P}_{s 20}$ at the measuring plane $P_{s 1}$ and $P_{s 2}$ at a balance speed, and control voltage signal $\vec{u}_{1}=u_{1} e^{j\left(\omega_{0} t+\varphi_{b 1}\right)}$ and $\vec{u}_{2}=u_{2} e^{j\left(\omega_{0} t+\varphi_{b 2}\right)}$ of the left and right active magnetic bearing were applied to balancing plane $P_{b 1}$ and plane $P_{b 2}$ respectively, and vibration displacement vectors at each measuring plane were obtained as $\vec{P}_{s 11}=P_{s 11} e^{j\left(\omega_{0} t+\varphi_{s 11}\right)}, \quad \vec{P}_{s 12}=P_{s 12} e^{j\left(\omega_{0} t+\varphi_{s 12}\right)}, \quad \vec{P}_{s 21}=P_{s 21} e^{j\left(\omega_{0} t+\varphi_{s 12}\right)} \quad$ and $\vec{P}_{s 22}=P_{s 22} e^{j\left(\omega_{0} t+\varphi_{s 22}\right)}$. The influence coefficient of the control voltage $\vec{u}_{1}$ on the displacement of the measuring plane $P_{s 1}$ and $P_{s 2}$ after the application of the control voltage $\vec{u}_{1}$ on the balancing plane $P_{b 1}$ is obtained, as shown in this Equation.

$$
\left[\begin{array}{ll}
\vec{a}_{11} & \vec{a}_{12} \\
\vec{a}_{21} & \vec{a}_{22}
\end{array}\right]=\left[\begin{array}{ll}
\frac{\vec{P}_{s 11}-\vec{P}_{s 10}}{\vec{u}_{1}} & \frac{\vec{P}_{s 21}-\vec{P}_{s 20}}{\vec{u}_{1}} \\
\frac{\vec{P}_{s 12}-\vec{P}_{s 10}}{\vec{u}_{2}} & \frac{\vec{P}_{s 22}-\vec{P}_{s 20}}{\vec{u}_{2}}
\end{array}\right]
$$

Assuming that the control voltage signal $\vec{U}_{1}=U_{1} e^{j\left(\omega_{0} t+\varphi_{1}\right)}$ is added to the balancing plane $P_{b 1}$ and the control voltage signal $\vec{U}_{2}=U_{2} e^{j\left(\omega_{0} t+\varphi_{2}\right)}$ is added to the balancing plane $P_{b 2}$, the displacement at the measuring plane $P_{s 1}$ and $P_{s 2}$ can be decreased to zero, the unbalanced vector equation can be obtained as shown in the equation.

$$
\left\{\begin{array}{l}
\vec{a}_{1} \vec{U}_{1}+\vec{a}_{2} \vec{U}_{2}+\vec{P}_{s 10}=0 \\
\vec{b}_{1} \vec{U}_{1}+\vec{b}_{2} \vec{U}_{2}+\vec{P}_{s 20}=0
\end{array}\right.
$$

By solving the above vector equation, the compensation voltage signals that should be added on the balancing plane $P_{b 1}$ and $P_{b 2}$ can be obtained respectively 
1st International Electronic Conference on Actuator Technology: Materials, Devices and Applications (IeCAT 2020)

$$
\vec{U}_{1}=U_{1} e^{j\left(\omega_{0} t+\varphi_{1}\right)}=\frac{\left|\begin{array}{cc}
-\vec{P}_{s 10} & \vec{a}_{2} \\
-\vec{P}_{s 20} & \vec{b}_{2}
\end{array}\right|}{\left|\begin{array}{ll}
\vec{a}_{1} & \vec{a}_{2} \\
\vec{b}_{1} & \vec{b}_{2}
\end{array}\right|} \quad \vec{U}_{2}=U_{2} e^{j\left(\omega_{0} t+\varphi_{2}\right)}=\frac{\left|\begin{array}{ll}
\vec{a}_{1} & -\vec{P}_{s 10} \\
\vec{b}_{1} & -\vec{P}_{s 20}
\end{array}\right|}{\left|\begin{array}{ll}
\vec{a}_{1} & \vec{a}_{2} \\
\vec{b}_{1} & \vec{b}_{2}
\end{array}\right|}
$$

\subsection{Principle of the online unbalanced vibration suppression}

The compensation voltage signal obtained by above formulation is the compensation signal of the equivalent unbalanced interference voltage signal $\frac{m e \omega^{2}}{X(\omega)} \angle \theta-\phi(\omega)$, with equal amplitude and opposite phase. From the above analysis, it can be seen that when the influence coefficient method based on the magnetic bearing is used to compensate for the unbalanced vibration of the rotor, the control signal can be used instead of the traditional shutdown and counterweight to suppress the unbalanced vibration of the magnetically suspended rotor, as shown in Fig. 3.

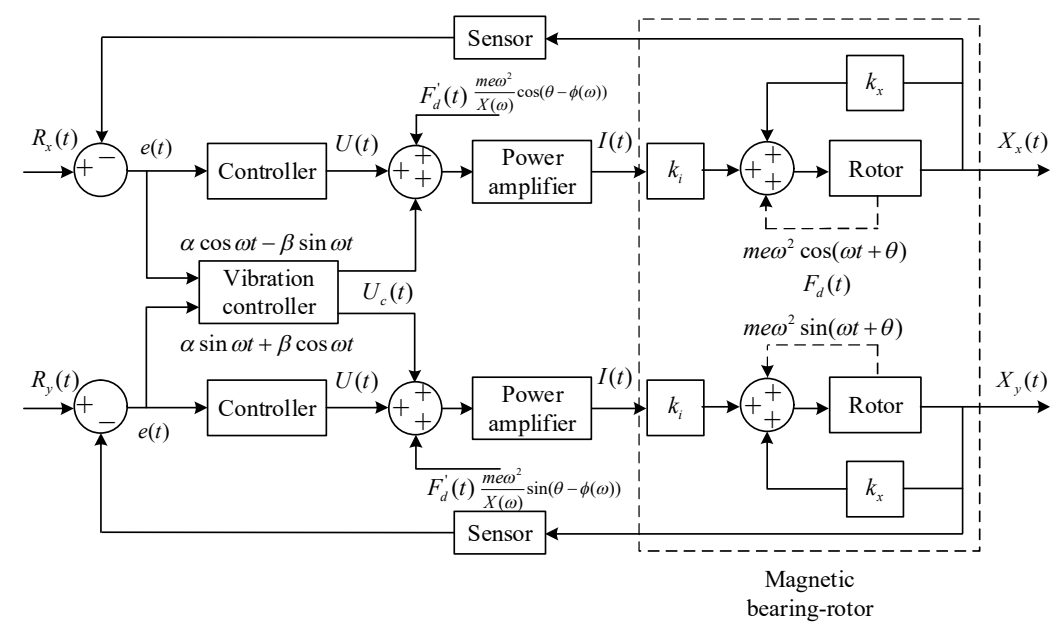

Figure 3. Principle of online unbalance compensation for maglev rotor.

\section{Results}

In order to verify the feasibility of the algorithm in this paper, an online rotor unbalance compensation experiment is carried out. The experimental device is shown in Fig. 4 and Fig. 5, and the rotor speed is set to $3000 \mathrm{r} / \mathrm{min}$. The algorithm in this paper is used to suppress the unbalanced vibration of the rotor at the left and right magnetic bearings respectively, and the results are shown in Fig. 6. The blue curve in the figure is the trajectory of the rotor axis before compensation, and the red curve is the trajectory of the axis after compensation.

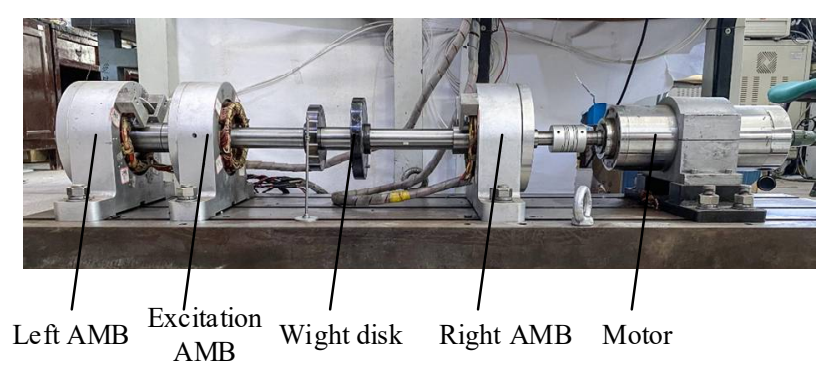

Figure 4. Maglev rotor experimental platform. 

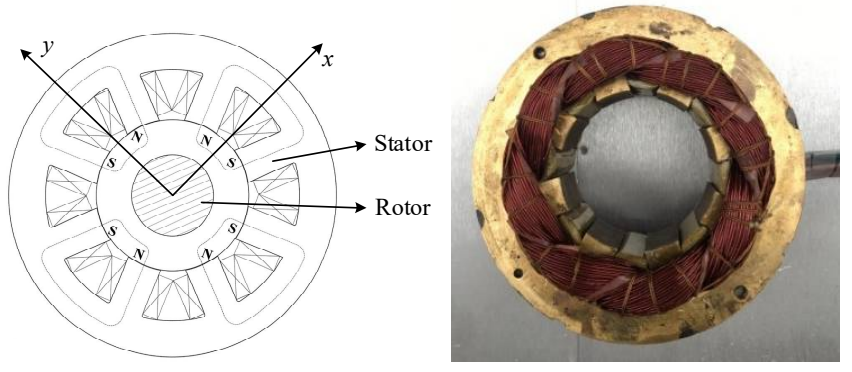

Figure 5. Active magnetic bearing.

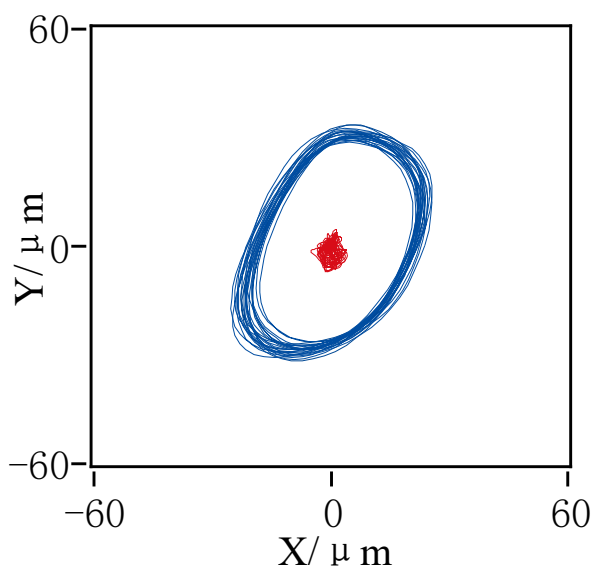

(a) Left active magnetic bearing

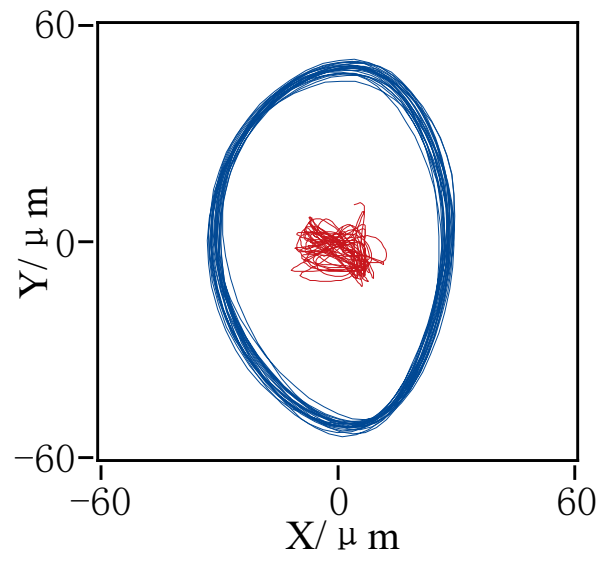

(b) Right active magnetic bearing

Figure 6. Track of rotor vibration with online unbalance compensation.

While the rotor speed is being held at $3000 \mathrm{r} / \mathrm{min}$, the original rotor unbalance vibrations in two AMBs are recorded, as shown in Fig. 6. The unbalanced vibration of the maglev rotor is greatly reduced. The maximum amplitude of the rotor vibration at the left magnetic bearing is reduced by $81.4 \%$, and the maximum amplitude of the rotor vibration at the right magnetic bearing is reduced by $77.3 \%$.

\section{Conclusions}

The simulations and experiments show that the proposed method achieves the good effectiveness to suppress the unbalanced vibration of rotor online. This paper uses electromagnetic force to try to apply control voltage to the flexible rotor supported by active magnetic bearing, instead of the traditional shutdown test and phase sensor measure. Use the influence coefficient method to detect the unbalanced quality information of the rotor with the mass unbalance, and obtain the magnitude of the compensation voltage. The cross-correlation method and feedback tracking control method in steady state is used to extract the fundamental frequency vibration signal of the rotor to obtain the phase information of the unbalanced vibration. The effectiveness of the algorithm in this paper is verified through experiments, and the unbalanced vibration of the rotor at the left and right magnetic bearings is reduced by $81.4 \%$ and $77.3 \%$.

\section{References}

1. Yefa Hu, Zude Zhou, Zhengfeng Jiang. Foundation Theory and Application of Active Magnetic Bearing. Beijing: China Machine Press, 2006.

2. Małgorzata Gizelska, Kozanecka D , Kozanecki Z . Monitoring and Diagnostics of the Rotating System with an Active Magnetic Bearing[J]. Solid State Phenomena, 2013, 198:547-552.

3. Jiang Kejian, Zhu Changsheng, and Chen Liangliang. Unbalance Compensation by Recursive Seeking Unbalance Mass Position in Active Magnetic Bearing-Rotor System[J]. IEEE TRANSACTIONS ON 
1st International Electronic Conference on Actuator Technology: Materials, Devices and Applications (IeCAT 2020) INDUSTRIAL ELECTRONICS, 2015:62(9):5655-5664.

4. $\mathrm{Xu}$ X , Fan P P . Rigid Rotor Dynamic Balancing by Two-Plane Correction with the Influence Coefficient Method[J]. Applied Mechanics \& Materials, 2013, 365-366:211-215.

5. Cui P , Liu Z, Xu H, et al. Harmonic Vibration Force Suppression of Magnetically Suspended Rotor With Frequency-Domain Adaptive LMS[J]. IEEE Sensors Journal, 2020, 20(3):1166-1175.

6. Ran S, Hu Y, Wu H, et al. Resonance Vibration Control for AMB Flexible Rotor System Based on $\mu-$ Synthesis Controller[J]. Mathematical Problems in Engineering, 2018, 2018(PT.17):4362101.14362101.16.

7. Chen Q, Liu G, Han B . Suppression of Imbalance Vibration in AMB-Rotor Systems Using Adaptive Frequency Estimator[J]. IEEE Transactions on Industrial Electronics, 2015, 62(12):7696-7705.

(C) 2020 by the authors; licensee MDPI, Basel, Switzerland. This article is an open access article distributed under the terms and conditions of the Creative Commons by Attribution (CC-BY) license (http://creativecommons.org/licenses/by/4.0/). 